\title{
The Additional Value of PET in the Assessment of Cerebral Small Vessel Disease
}

\author{
Wolf-Dieter Heiss \\ Max Planck Institute for Metabolism Research, Cologne, Germany
}

The diagnosis of cerebral small vessel disease (SVD) is difficult because there is no consensus on clinical criteria, and therefore imaging is important for diagnosis. Most patients undergo brain imaging by CT, which is able to detect ischemic strokes, hemorrhages, and brain atrophy and may also indicate white matter changes. MRI remains the key neuroimaging modality and is preferred to $\mathrm{CT}$ in vascular cognitive impairment ( $\mathrm{VCl}$ ) because it has higher sensitivity and specificity for detecting pathologic changes. These modalities for imaging morphology permit detection of vascular lesions traditionally attributed to $\mathrm{VCl}$ in subcortical areas of the brain, single infarction or lacunes in strategic areas (thalamus or angular gyrus), or large cortical-subcortical lesions reaching a critical threshold of tissue loss. In SVD, multiple punctuate or confluent lesions can be seen in the white matter by $\mathrm{MRI}$ and were called leukoaraiosis. Another major neuroimaging finding of SVD in $\mathrm{VCl}$ are microhemorrhages. However, whereas $\mathrm{CT}$ and $\mathrm{MRI}$ are able to detect morphologic lesions, these modalities cannot determine functional consequences of the underlying pathologic changes. PET can support the clinical diagnosis by visualizing cerebral functions in typically affected brain regions. In SVD, ${ }^{18} \mathrm{~F}-\mathrm{FDG}$ PET can clearly differentiate scattered areas of focal cortical and subcortical hypometabolism that differ from the typical metabolic pattern seen in Alzheimer dementia (AD) with marked hypometabolism affecting the association areas. Additional PET tracers can further support the diagnosis of a type of dementia and also yield information on the underlying pathophysiology

Key Words: cerebral small vessel disease; SVD; vascular cognitive impairment; vascular dementia; neuroimaging; MRI; CT/PET

J Nucl Med 2018; 59:1660-1664

DOI: 10.2967/jnumed.118.214270

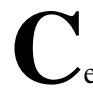

erebral small vessel disease (SVD) is an umbrella term covering a variety of abnormalities affecting the smallest cerebral blood vessels, including perforating ateriolis, capillaries, and venules (1). The most common etiology of SVD is hypertensive artheropathy characterized by collagenus thickening of the vessel wall with the usual risk factors hypertension, diabetes mellitus, dyslipidemia, cigarette smoking, and age (2). A more sporadic cause is cerebral amyloid angiopathy, a degenerative disorder with

Received May 7, 2018; revision accepted Jun. 11, 2018.

For correspondence or reprints contact: Wolf-Dieter Heiss, Max Planck Institute for Metabolism Research, Gleueler Strasse 50, D-50931 Cologne, Germany.

E-mail: wdh@sf.mpg.de

Published online Jun. 29, 2018.

COPYRIGHT (C 2018 by the Society of Nuclear Medicine and Molecular Imaging. progressive deposition of amyloid- $\beta$ in the media and adventitia of small arteries, arterioles, and sometimes capillaries in the cerebral cortex (3). Other rare causes include inherited genetic SVD (CADASIL, MELAS, Fabry's disease), inflammatory-immunologic mediated SVD (systemic lupus erythematodes, scleroderma, ANCA-associated SVD, nervous system vasculitides secondary to infections), venous collagenosis, and postradiation angiopathy (4).

In many cases, cerebral SVD seems to be a consequence of atherosclerosis affecting the smaller blood vessels that nourish brain tissue. Just as one's larger blood vessels in the heart or elsewhere can accumulate plaque, inflammation, and chronic damage over the years, so can the smaller blood vessels. Such chronic damage can lead the small blood vessels in the brain to become blocked (which starves brain cells of oxygen, and which we technically call ischemia), or to leak (which causes bleeding, which we call hemorrhage and can damage nearby brain cells). When little bits of brain get damaged in these ways, they can change appearance on radiologic scans. So when an MRI report says "white matter changes," this means the radiologist is seeing signs of cerebral SVD (5). Specific pathologic changes of cerebral SVD include lacunar infarcts (which are a type of small stroke), white matter hyperintensities (which are a radiologic finding), and cerebral microbleeds (which means bleeding in the brain from a very small blood vessel) (6). Such signs of SVD may be described as mild, moderate, or severe/extensive, depending on how widespread they are (7). The severity of symptoms tends to correspond to whether radiologic imaging shows the cerebral SVD to be mild, moderate, or severe. Many older adults with cerebral SVD will have no noticeable symptoms. This is sometimes called silent SVD (8).

Many clinical symptoms have been associated with cerebral SVD, especially when it is moderate or severe. These include:

- Cognitive impairment: SVD is especially causing deficits in executive function, attention, verbal fluency, and set-shifting. Several studies (9) have found that cerebral SVD is correlated with worse scores on the Mini-Mental State Examination. When problems with thinking skills are associated with SVD, this can be called "vascular cognitive impairment" (VCI).

- Problems with walking and balance: white matter lesions have been repeatedly associated with gait disturbances, frontal gait abraxia, and mobility difficulties. A study (10) found that moderate or severe cerebral SVD was associated with a decline in gait and balance function.

- Strokes: a metaanalysis (1) concluded that white matter hyperintensities are associated with a more than 2-fold increase in the risk of stroke.

- Depression: white matter changes have been associated with a higher risk of depression in older people and may represent 
a contributor to depression that is particular to having firsttime depression in later life (11).

- Vascular dementia (VaD): signs of cerebral SVD are associated with both having $\mathrm{VaD}$ and eventually developing $\mathrm{VaD}(1)$.

- Other dementias: research (12) suggests that cerebral SVD is also associated with an increased risk-or increased severityof other forms of dementia, such as Alzheimer disease (AD). Autopsy studies have confirmed that many older adults with dementia show signs of both Alzheimer pathology and cerebral SVD.

- Transition to disability or death: in a study (7) of 639 nondisabled older persons (mean age, 74), over a 3-y follow-up period, $29.5 \%$ of participants with severe white matter changes and $15.1 \%$ of participants with moderate white matter changes developed disabilities or died. In comparison, only $10.5 \%$ of participants with mild white matter changes transitioned to disability or death over $3 \mathrm{y}$. The researchers concluded that severity of cerebral SVD is an important risk factor for overall decline in older adults.

Recently, consensus diagnostic criteria of subcortical SVD have been published (13) and also the imaging standards for research have been determined (14). It is important to realize SVD of the brain primarily affects cognitive and emotional domains and usually does not lead to sudden and dramatic motor impairments as is seen in large vessel occlusions of the brain. Since clinical signs and symptoms are often insufficient to allow for a final diagnosis and usually cannot differentiate among the various etiologies, neuroimaging plays an important role in the management of patients with impaired cognition. Neuroimaging is also relevant for the detection of sporadic SVD (15). This review will concentrate on the importance of imaging procedures for the diagnosis of SVD as a cause of VCI. Additionally, it will describe the value of molecular and functional imaging for the detection of the relationship of morphologic damage and cognitive impairment and of the differentiation between vascular and degenerative causes of dementia.

\section{IMAGING MORPHOLOGIC SUBSTRATES OF SVD}

Neuroimaging provides important information on neuroanatomical substrates of the disorder, plays an important role in the diagnosis, and adds to prediction of VaD. Whereas CT is still the leading imaging study in the management of acute stroke, MRI remains the key neuroimaging modality in $\operatorname{VaD}(16,17)$. If not contraindicated, MRI, rather than CT, is preferred for research and routine clinical use because it has higher sensitivity and specificity for detecting pathologic changes. Standards for neuroimaging with a widely accepted terminology permitting comparison of findings between centers have been recommended (STandards for ReportIng Vascular changes on Neuroimaging [STRIVE]) (14). Numerous studies identified MRI markers of SVD (lacunes, white matter hyperintensities, cerebral microbleeds, silent infarcts, white matter changes, global cerebral atrophy, medial-temporal lobe atrophy) as determinants of $\mathrm{VaD}(18,19)$. Vascular lesions traditionally attributed to VCI comprise subcortical areas of the brain, especially subfrontal white matter circuits, strategic areas of single infarction such as the dominant thalamus or angular gyrus, deep frontal areas and the left hemisphere, and bilateral brain infarcts or volume-driven cortical-subcortical infarctions reaching a critical threshold of tissue loss or injury (20). Recently, enlargements of perivascular spaces were identified as MRI markers of SVD. These are associated with the pathogenesis of vascular-related cognitive impairment in older individuals (21). The burden of SVD as a risk of stroke is increased in type 2 diabetes (22).

SVD identified on MRI in the white matter is called leukoaraiosis (23). Leukoaraiosis presents as multiple punctuate or confluent lesions, but more often as incomplete infarcts, and is commonly seen in healthy elderly (24) and in subjects with migraines. The markers of SVD-white matter hyperintensities, lacunes, dilated vascular spaces, microbleeds, and brain volumeare related to a decrease in regional cerebral blood flow (25) and must be clearly defined to be reliably used for the diagnosis of this vascular disorder and its progression (18). Some studies have suggested that to assess in single cases how much the lesion load affects cognition, a threshold of $10 \mathrm{~cm}^{2}(26)$ or $25 \%$ of total white matter (27) is required before $\mathrm{VaD}$ is detectable clinically. On fluid attenuated inversion recovery images, incomplete infarcts present as hyperintensities, whereas complete infarcts present as lacunae, which are hypointense in relation to the brain and isointense to the cerebrospinal fluid. Diffusion-weighted MRI is best suited to reveal surrogate markers of SVD progression (28). After stroke, medial temporal lobe atrophy is related to cognitive impairment rather than markers of SVD (29).

Microhemorrhages are the third major neuroimaging aspect of SVD, and in 1 study they were found in $65 \%$ of $\mathrm{VaD}$ cases $(30)$. Although macrohemorrhages associated with cognitive impairment (e.g., venous infarcts) can be seen on conventional T1- and T2weighted spin echo images, microhemorrhages often cannot be seen in these sequences, but can be detected accurately using $\mathrm{T} 2 *$-weighted gradient echo images. Microhemorrhages are also a defining feature of cerebral amyloid angiopathy (31).

Morphologic imaging indicates the extent and severity of SVD, but the observed changes are not directly related to functional or cognitive impairment and prognosis. In 1 study (32), several MRI findings together with older age and lower gait speed were identified as risk factors associated with mortality. Functional effects of SVD can be detected only by combining morphologic imaging with more complex technologies, for example, MR spectroscopy for showing biochemical changes (33) and functional MRI for analysis of cortical dysfunction (34). Advanced imaging techniques including molecular imaging by PET will help to understand the mechanisms by which SVD causes VCI and will improve diagnostic accuracy; they might reflect disease progression and in the future might be useful for development of therapies $(4,35)$.

\section{MOLECULAR IMAGING IN DIAGNOSIS OF DEMENTIA DUE TO SVD}

The diagnosis of VCI is difficult because there is no consensus on clinical criteria. Additionally, cerebral arteriosclerosis frequently is present in elderly patients and even small infarcts or white matter lesions occur in elderly subjects without either cognitive impairment or degenerative dementia. There is a tendency to diagnose VCI on the basis of MRI, which has a high sensitivity for white matter hyperintensities, which may be seen in normal elderly as well as those with VCI. Pathologic studies reveal a high incidence of both vascular and degenerative pathology of the Alzheimer type. There is a continuum from pure vascular over mixed to pure AD pathology $(36,37)$, which may lead to diagnostic confusion when only the MRI is used, and there is mixed pathology. PET provides additional information, which increases the diagnostic certainty.

PET can support the clinical diagnosis by visualizing cerebral functions in typically affected brain regions. PET of ${ }^{18}$ F-FDG for 


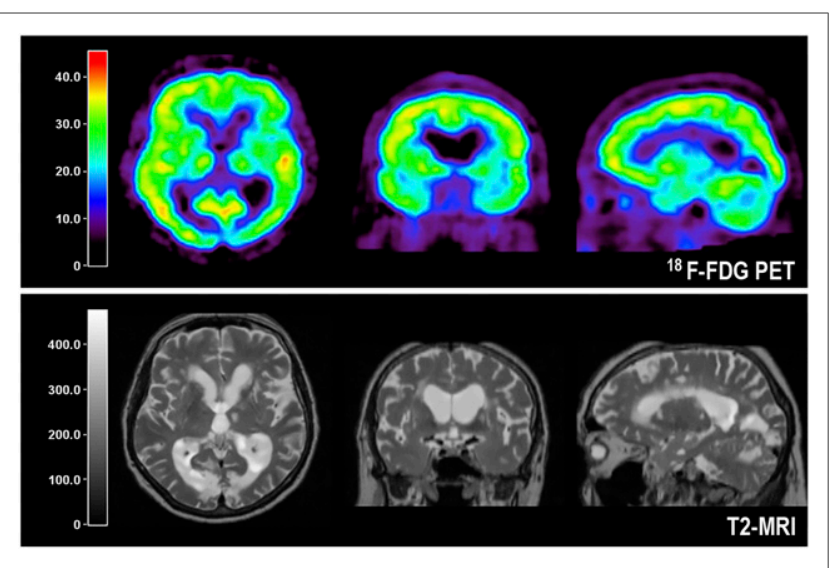

FIGURE 1. Coregistered T2-weighted MRI and PET orthogonal slices through brain of patient with SVD. T2-MRI shows typical periventricular and multiple small white matter lesions, and PET images reveal widespread effect of these lesions on glucose metabolism of whole cortex and basal ganglia causing $\mathrm{VCl}$.

measurement of regional cerebral glucose metabolism (rCMRglc) has shown a typical metabolic pattern in patients with probable AD: hypometabolism in temporoparietal and frontal association areas, but relative recessing of primary cortical areas, basal ganglia, and cerebellum. In VCI, a different pattern is seen (38): in VCI ${ }^{18}$ F-FDG PET can clearly differentiate scattered areas of focal cortical and subcortical hypometabolism (Fig. 1) that differ from the typical metabolic pattern seen in AD.

In VaD patients a significant reduction of rCMRglc in comparison to normal patients was observed in widespread cerebral regions (middle frontal cortex, temporoparietal cortex, basal ganglia, cerebellum, and brain stem). In subcortical areas and primary sensorimotor cortex this hypometabolism was more marked than

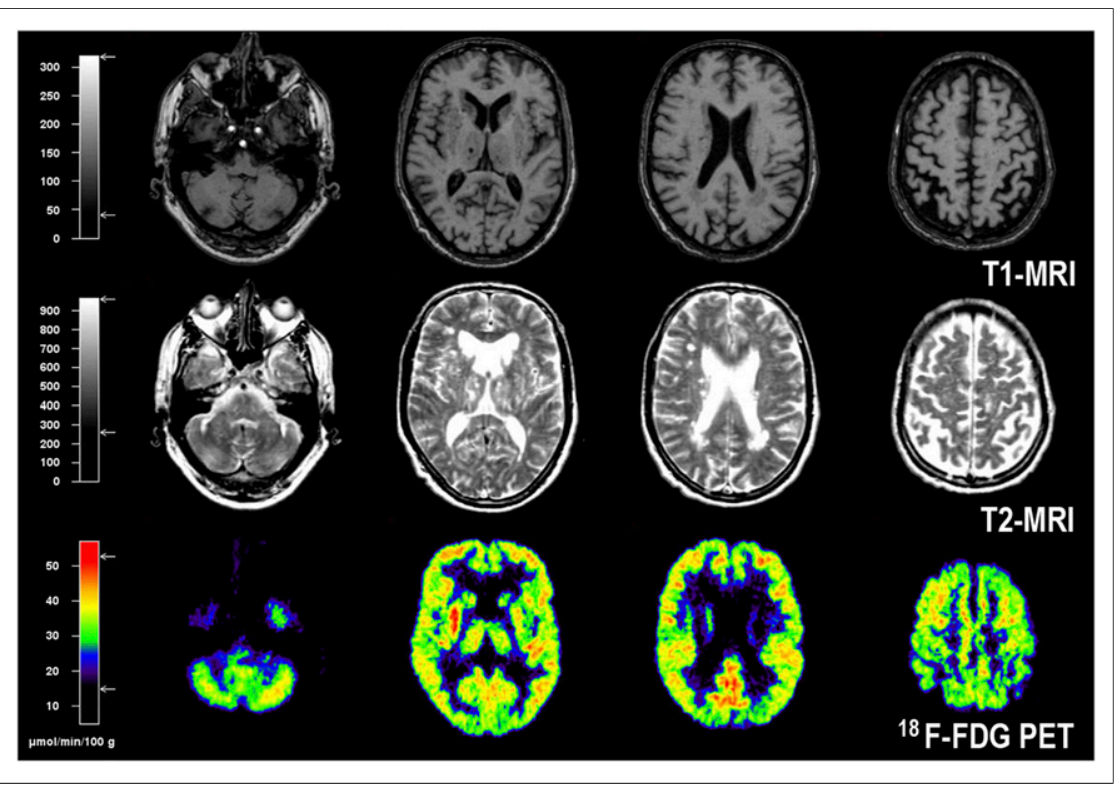

FIGURE 2. T1- and T2-weighted MR and PET images in VCl caused by multiple small infarcts, lacunes, and widespread white matter changes. PET images show diffuse impairment of metabolism in both hemispheres and also in basal ganglia, thalamus, and cerebellum. in $\mathrm{AD}$ whereas the association areas were less affected than in $\mathrm{AD}$. A metabolic ratio (rCMRglc of association areas divided by rCMRglc of primary areas, basal ganglia, cerebellum and brain stem) mainly reflecting the contrast between association areas and subcortical regions was significantly lower in $\mathrm{AD}$ than in $\mathrm{VCI}$ (39). Whereas it was not possible to identify a single region that could discriminate between VCI and AD, the composite pattern, as expressed in the metabolic ratio, was significantly different. Considering that the VCI patients in that study had mainly white matter hyperintensities and small subcortical infarcts, it suggests furthermore that even small infarcts in combination with white matter hyperintensities may contribute to cognitive decline. Rather than the total volume of infarction, the volume of functional tissue loss is more important, since it also includes the effects of incompletely infarcted tissue and morphologically intact but deafferented cortex (Fig. 2). Subcortical ischemic vascular disease can be distinguished from clinically probable $\mathrm{AD}$ by a more diffuse pattern of hypometabolism involving also the primary cortices, basal ganglia, thalamus and cerebellum.

\section{DIFFERENTIAL DIAGNOSIS OF DEMENTIAS WITH ${ }^{18}$ F-FDG PET}

Regional changes in cortical glucose metabolism are a finding indicating cognitive impairment; MRI features of SVD without regional changes in cortical glucose metabolism are a criterion to rule out VCI. AD is characterized by regional impairment of cerebral glucose metabolism in neocortical association areas, whereas the primary visual and sensorimotor cortex, basal ganglia, and cerebellum are relatively well preserved. In a multicenter study comprising 10 PET centers that used an automated voxelbased analysis of ${ }^{18}$ F-FDG PET images, the distinction between controls and $\mathrm{AD}$ patients had $93 \%$ sensitivity and $93 \%$ specificity (40). Significantly abnormal metabolism in mild cognitive impairment (MCI) indicates a high risk to develop dementia within the next 2 y. Reduced neocortical glucose metabolism can probably be detected with ${ }^{18} \mathrm{~F}$-FDG PET in AD on average 1 y before onset of subjective cognitive impairment (41).

Characteristic patterns of regional hypometabolism are also seen in other degenerative dementias. Frontotemporal dementia clinically characterized by changes in personality and behavior, semantic deficits, and progressive aphasia can be identified by distinct frontal or frontotemporal metabolic impairments that are typically quite asymmetrically centered in the frontolateral cortex and the anterior pole of the temporal lobe. Dementia with Lewy bodies (42), combining fluctuating consciousness, Parkinsonian symptoms, and impairment of visual perception including hallucinations, shows reduction of glucose metabolism in the primary visual cortex in addition to that in the posterior association areas. Other degenerative disorders show typical hypometabolism in the specifically affected brain structures: the putamen and cortex in corticobasal degeneration (43), the caudate nucleus in Huntington's chorea (44), the frontal cortex and midbrain in progressive 
supranuclear palsy (45), and the pons and cerebellum in olivopontocerebellar atrophy (46). In addition, depressive disorders may mimic cognitive impairment; in these cases, glucose metabolism does not show regional abnormalities.

\section{IMAGING SYNAPTIC TRANSMISSION AND ACCUMULATION OF PATHOLOGIC PROTEINS}

Additional PET tracers can further support the diagnosis of a type of dementia and also yield information on the underlying pathophysiology. Tracers permit the study of selectively affected transmitter/receptor systems, for example, the cholinergic system in $\mathrm{AD}$ - significant reduction of cholinergic activity in the cortex of $\mathrm{AD}$ patients and those with MCI and early conversion to $\mathrm{AD}$ (47) — or the dopaminergic system in dementia with Lewy bodies (48). Furthermore, tracers enable the detection of pathogenetic depositions, for example, amyloid and tau in AD (49). Particularly, the imaging of accumulation of pathologic proteins is a recent strategy to differentiate degenerative dementias: amyloid is a pathogenetic product in the development of AD and its accumulation is a key finding in this disease. Its accumulation can be imaged by ${ }^{11} \mathrm{C}$-labeled Pittsburgh compound B $\left({ }^{11} \mathrm{C}-\mathrm{PiB}\right)(50)$ or by several newer ${ }^{18} \mathrm{~F}$-labeled tracers $(51)$. Whereas only small amounts of amyloid can be detected in the white matter in normal aging (52), accumulation is visible in the frontal and temporoparietal cortex in AD and MCI (53). However, also in $20 \%-30 \%$ of aged persons without relevant cognitive impairment an increased accumulation of amyloid can be detected (54), and the grade of amyloid deposition as detected by PET is not related to the severity of cognitive impairment (55). That means that amyloid might be deposited in the brain eventually long before cognitive impairment is recognized. Amyloid deposition in combination with neuroinflammation as expressed in microglia activation might play a role in the development of poststroke dementia (56). Cortical amyloid deposition in combination with relevant MRI features of SVD is a diagnostic hint for mixed dementia.

A more specific pathologic protein produced in $\mathrm{AD}$ is tau, and its deposition in the mesial temporal lobe is an early marker of $\mathrm{AD}$ or MCI (57), and the amount of tau detected in the cortex by selective PET tracers is related to the severity of cognitive impairment (58). These PET tracers also detect the primary pathologic substrate in other degenerative dementias (e.g., tau in frontotemporal dementia) (59) and permit the differentiation between $\mathrm{AD}$ and VCI and other degenerative dementias (60-65).

The unique potentials of PET in localizing and quantifying metabolic changes in gray matter structures responsible for functional/cognitive disturbances and caused by SVD mainly affecting the white matter makes this technique the preferred tool for studying patients with VCI; the additional ability of PET to detect pathologic proteins (amyloid and tau) and to image synaptic transmission and receptor activity is of special value for the differential diagnosis of different types of dementia (62). All together, PET studies may shed light into the pathophysiologic mechanisms responsible for the disease in a single patient, and repeated investigations in the course may reflect progression and may help to demonstrate efficiency in the development of new treatment strategies (35). However, because of the scarcity of complete PET installations including cyclotron and radiochemistry, PET is mainly restricted to applications in research. With the installation of more clinical PET cameras as satellites to a central ${ }^{18} \mathrm{~F}-\mathrm{FDG}$-producing unit-a concept that is already successful for imaging in oncology—more patients with MCI could benefit from improved diagnostic imaging.

\section{CONCLUSION}

SVD denotes a group of diseases that affect the small vessels of the brain, mostly because of chronic hypertensive damage to small arteries and arterioles. The diagnosis of SVD is difficult because there is no consensus on clinical criteria. Clinical manifestations include cognitive impairment and emotional disturbances. The initially observed mild neurocognitive disorder usually is progressive and affects other domains such as sensorimotor functions, coordination, language, and memory. Over time it can result in vascular dementia. SVD is more frequent than previously thought and can now be better detected due to progress in neuroimaging. MRI remains the key neuroimaging modality in chronic VCI because it has higher sensitivity and specificity for detecting pathologic changes. It permits the detection of vascular lesions traditionally attributed to SVD in subcortical areas of the brain and lacunes in strategic areas (thalamus or angular gyrus). Multiple punctuates or confluent lesions can be seen in the white matter by MRI and are called leukoaraiosis. Another major neuroimaging finding of SVD in VCI are microhemorrhages. However, whereas CT and MRI are able to detect morphologic lesions, these modalities cannot determine functional consequences of the underlying pathologic changes, which can be detected and quantitatively assessed by PET. Additionally, pathologic studies reveal a high incidence of mixed dementias with both vascular and degenerative pathology of the Alzheimer type. This leads to diagnostic confusion when only MRI is used. That means that molecular imaging by PET may play an important role in the differentiation of vascular and degenerative cognitive impairment.

\section{DISCLOSURE}

No potential conflict of interest relevant to this article was reported.

\section{REFERENCES}

1. Debette S, Markus HS. The clinical importance of white matter hyperintensities on brain magnetic resonance imaging: systematic review and meta-analysis. BMJ. 2010;341:c3666.

2. Pantoni L. Cerebral small vessel disease: from pathogenesis and clinical characteristics to therapeutic challenges. Lancet Neurol. 2010;9:689-701.

3. Charidimou A, Gang Q, Werring DJ. Sporadic cerebral amyloid angiopathy revisited: recent insights into pathophysiology and clinical spectrum. J Neurol Neurosurg Psychiatry. 2012;83:124-137.

4. Banerjee G, Wilson D, Jager HR, Werring DJ. Novel imaging techniques in cerebral small vessel diseases and vascular cognitive impairment. Biochim Biophys Acta. 2016;1862:926-938.

5. Smith EE, O'Donnell M, Dagenais G, et al. Early cerebral small vessel disease and brain volume, cognition, and gait. Ann Neurol. 2015;77:251-261.

6. Mok V, Kim JS. Prevention and management of cerebral small vessel disease. J Stroke. 2015;17:111-122.

7. Inzitari D, Pracucci G, Poggesi A, et al. Changes in white matter as determinant of global functional decline in older independent outpatients: three year followup of LADIS (leukoaraiosis and disability) study cohort. BMJ. 2009;339:b2477.

8. Vermeer SE, Longstreth WT Jr, Koudstaal PJ. Silent brain infarcts: a systematic review. Lancet Neurol. 2007;6:611-619.

9. van der Flier WM, van Straaten EC, Barkhof F, et al. Small vessel disease and general cognitive function in nondisabled elderly: the LADIS study. Stroke. 2005; 36:2116-2120.

10. Kreisel SH, Blahak C, Bazner H, et al. Deterioration of gait and balance over time: the effects of age-related white matter change-the LADIS study. Cerebrovasc Dis. 2013;35:544-553.

11. Herrmann LL, Le Masurier M, Ebmeier KP. White matter hyperintensities in late life depression: a systematic review. J Neurol Neurosurg Psychiatry. 2008;79:619-624. 
12. Gorelick PB, Scuteri A, Black SE, et al. Vascular contributions to cognitive impairment and dementia: a statement for healthcare professionals from the American Heart Association/American Stroke Association. Stroke. 2011;42:2672-2713.

13. Rosenberg GA, Wallin A, Wardlaw JM, et al. Consensus statement for diagnosis of subcortical small vessel disease. J Cereb Blood Flow Metab. 2016;36:6-25.

14. Wardlaw JM, Smith EE, Biessels GJ, et al. Neuroimaging standards for research into small vessel disease and its contribution to ageing and neurodegeneration. Lancet Neurol. 2013;12:822-838.

15. Charidimou A, Pantoni L, Love S. The concept of sporadic cerebral small vessel disease: a road map on key definitions and current concepts. Int J Stroke. 2016;11: 6-18.

16. Vitali P, Migliaccio R, Agosta F, Rosen HJ, Geschwind MD. Neuroimaging in dementia. Semin Neurol. 2008;28:467-483.

17. Zwanenburg JJM, van Osch MJP. Targeting cerebral small vessel disease with MRI. Stroke. 2017;48:3175-3182.

18. De Guio F, Jouvent E, Biessels GJ, et al. Reproducibility and variability of quantitative magnetic resonance imaging markers in cerebral small vessel disease. J Cereb Blood Flow Metab. 2016;36:1319-1337.

19. Shibuya M, Leite CDC, Lucato LT. Neuroimaging in cerebral small vessel disease: Update and new concepts. Dement Neuropsychol. 2017;11:336-342.

20. Peres R, De Guio F, Chabriat H, Jouvent E. Alterations of the cerebral cortex in sporadic small vessel disease: a systematic review of in vivo MRI data. J Cereb Blood Flow Metab. 2016;36:681-695.

21. Ding J, Sigurethsson S, Jonsson PV, et al. Large perivascular spaces visible on magnetic resonance imaging, cerebral small vessel disease progression, and risk of dementia: the age, gene/environment susceptibility-Reykjavik study. JAMA Neurol. 2017;74:1105-1112.

22. Umemura T, Kawamura T, Hotta N. Pathogenesis and neuroimaging of cerebral large and small vessel disease in type 2 diabetes: a possible link between cerebral and retinal microvascular abnormalities. J Diabetes Investig. 2017;8:134-148.

23. Hachinski VC, Potter P, Merskey H. Leuko-araiosis. Arch Neurol. 1987;44:21-23.

24. de Leeuw FE, de Groot JC, Achten E, et al. Prevalence of cerebral white matter lesions in elderly people: a population based magnetic resonance imaging study. The Rotterdam Scan Study. J Neurol Neurosurg Psychiatry. 2001;70:9-14.

25. Shi Y, Thrippleton MJ, Makin SD, et al. Cerebral blood flow in small vessel disease: a systematic review and meta-analysis. J Cereb Blood Flow Metab. 2016;36: $1653-1667$

26. Boone KB, Miller BL, Lesser IM, et al. Neuropsychological correlates of whitematter lesions in healthy elderly subjects: a threshold effect. Arch Neurol. 1992;49: 549-554.

27. van Straaten EC, Scheltens P, Knol DL, et al. Operational definitions for the NINDS-AIREN criteria for vascular dementia: an interobserver study. Stroke. 2003;34:1907-1912.

28. Lyoubi-Idrissi AL, Jouvent E, Poupon C, Chabriat H. Diffusion magnetic resonance imaging in cerebral small vessel disease. Rev Neurol (Paris). 2017;173:201-210.

29. Arba F, Quinn T, Hankey GJ, et al. Cerebral small vessel disease, medial temporal lobe atrophy and cognitive status in patients with ischaemic stroke and transient ischaemic attack. Eur J Neurol. 2017;24:276-282.

30. Cordonnier C, van der Flier WM, Sluimer JD, Leys D, Barkhof F, Scheltens P. Prevalence and severity of microbleeds in a memory clinic setting. Neurology. 2006;66:1356-1360.

31. Farid K, Charidimou A, Baron JC. Amyloid positron emission tomography in sporadic cerebral amyloid angiopathy: a systematic critical update. Neuroimage Clin. 2017; 15:247-263.

32. van der Holst HM, van Uden IW, Tuladhar AM, et al. Factors associated with 8-year mortality in older patients with cerebral small vessel disease: The Radboud University Nijmegen Diffusion Tensor and Magnetic Resonance Cohort (RUN DMC) Study. JAMA Neurol. 2016;73:402-409.

33. Sappey-Marinier D, Calabrese G, Hetherington HP, et al. Proton magnetic resonance spectroscopy of human brain: applications to normal white matter, chronic infarction, and MRI white matter signal hyperintensities. Magn Reson Med. 1992;26:313-327.

34. Schaefer A, Quinque EM, Kipping JA, et al. Early small vessel disease affects frontoparietal and cerebellar hubs in close correlation with clinical symptoms: a resting-state fMRI study. J Cereb Blood Flow Metab. 2014;34:1091-1095.

35. Bath PM, Wardlaw JM. Pharmacological treatment and prevention of cerebral small vessel disease: a review of potential interventions. Int J Stroke. 2015;10:469-478.

36. Román G. Diagnosis of vascular dementia and Alzheimer's disease. Int J Clin Pract. 2001;120:9-13.
37. Ashraf GM, Chibber S, Mohammad, et al. Recent updates on the association between Alzheimer's disease and vascular dementia. Med Chem. 2016;12:226-237.

38. Heiss WD, Zimmermann-Meinzingen S. PET imaging in the differential diagnosis of vascular dementia. J Neurol Sci. 2012;322:268-273.

39. Mielke R, Heiss WD. Positron emission tomography for diagnosis of Alzheimer's disease and vascular dementia. J Neural Transm Suppl. 1998;53:237-250.

40. Herholz K, Salmon E, Perani D, et al. Discrimination between Alzheimer dementia and controls by automated analysis of multicenter FDG PET. Neuroimage. 2002;17:302-316.

41. Chételat G, Desgranges B. de ISV, Viader F, Eustache F, Baron JC. Mild cognitive impairment: can FDG-PET predict who is to rapidly convert to Alzheimer's disease? Neurology. 2003;60:1374-1377.

42. Bohnen NI, Muller M, Frey KA. Molecular imaging and updated diagnostic criteria in Lewy Body dementias. Curr Neurol Neurosci Rep. 2017;17:73.

43. Murgai AA, Jog MS. Neurophysiology and neurochemistry of corticobasal syndrome. J Neurol. 2018.

44. Ciarmiello A, Giovacchini G, Giovannini E, et al. Molecular imaging of Huntington's disease. J Cell Physiol. 2018;265:991-998.

45. Whitwell JL, Hoglinger GU, Antonini A, et al. Radiological biomarkers for diagnosis in PSP: where are we and where do we need to be? Mov Disord. 2017;32: 955-971.

46. Gilman S. Functional imaging with positron emission tomography in multiple system atrophy. J Neural Transm. 2005;112:1647-1655.

47. Herholz K, Weisenbach S, Kalbe E, Diederich NJ, Heiss WD. Cerebral acetylcholine esterase activity in mild cognitive impairment. Neuroreport. 2005;16: 1431-1434.

48. Hilker R, Thomas AV, Klein JC, et al. Dementia in Parkinson disease: functional imaging of cholinergic and dopaminergic pathways. Neurology. 2005;65:1716-1722.

49. Braak H, Braak E. Neuropathological staging of Alzheimer related changes. Acta Neuropathol (Berl). 1991;82:239-259.

50. Klunk WE, Engler H, Nordberg A, et al. Imaging brain amyloid in Alzheimer's disease with Pittsburgh compound-B. Ann Neurol. 2004;55:306-319.

51. Villemagne VL, Mulligan RS, Pejoska S, et al. Comparison of ${ }^{11} \mathrm{C}-\mathrm{PiB}$ and ${ }^{18} \mathrm{~F}-$ florbetaben for Abeta imaging in ageing and Alzheimer's disease. Eur J Nucl Med Mol Imaging. 2012;39:983-989.

52. Aizenstein HJ, Nebes RD, Saxton JA, et al. Frequent amyloid deposition without significant cognitive impairment among the elderly. Arch Neurol. 2008;65:15091517.

53. Barthel H, Schroeter ML, Hoffmann KT, Sabri O. PET/MR in dementia and other neurodegenerative diseases. Semin Nucl Med. 2015;45:224-233.

54. Herholz K, Ebmeier K. Clinical amyloid imaging in Alzheimer's disease. Lancet Neurol. 2011;10:667-670.

55. Yotter RA, Doshi J, Clark V, et al. Memory decline shows stronger associations with estimated spatial patterns of amyloid deposition progression than total amyloid burden. Neurobiol Aging. 2013;34:2835-2842.

56. Thiel A, Cechetto DF, Heiss WD, Hachinski V, Whitehead SN. Amyloid burden, neuroinflammation, and links to cognitive decline after ischemic stroke. Stroke. 2014;45:2825-2829.

57. Maruyama M, Shimada H, Suhara T, et al. Imaging of tau pathology in a tauopathy mouse model and in Alzheimer patients compared to normal controls. Neuron. 2013;79:1094-1108.

58. Small GW, Bookheimer SY, Thompson PM, et al. Current and future uses of neuroimaging for cognitively impaired patients. Lancet Neurol. 2008;7:161-172.

59. Spillantini MG, Goedert M. Tau pathology and neurodegeneration. Lancet Neurol. 2013;12:609-622.

60. Schöll M, Lockhart SN, Schonhaut DR, et al. PET imaging of tau deposition in the aging Human brain. Neuron. 2016;89:971-982.

61. Sepulcre J, Schultz AP, Sabuncu M, et al. In vivo tau, amyloid, and gray matter profiles in the aging brain. J Neurosci. 2016;36:7364-7374.

62. Drzezga A, Barthel H, Minoshima S, Sabri O. Potential clinical applications of PET/MR imaging in neurodegenerative diseases. J Nucl Med. 2014;55:47S-55S.

63. Chiotis K, Saint-Aubert L, Savitcheva I, et al. Imaging in-vivo tau pathology in Alzheimer's disease with THK5317 PET in a multimodal paradigm. Eur J Nucl Med Mol Imaging. 2016;43:1686-1699.

64. Choi Y, Ha S, Lee YS, Kim YK, Lee DS, Kim DJ. Development of tau PET imaging ligands and their utility in preclinical and clinical studies. Nucl Med Mol Imaging. 2018;52:24-30.

65. Villemagne VL, Dore V, Burnham SC, Masters CL, Rowe CC. Imaging tau and amyloid-beta proteinopathies in Alzheimer disease and other conditions. Nat Rev Neurol. 2018;14:225-236. 